\title{
Preconditioning with netrin-1 in Acute myocardial infarction model of rat improves systolic and diastolic left ventricular dysfunction
}

\author{
J. H. Ryu ${ }^{1}$, S. Lee ${ }^{1}$, S. H. Kang ${ }^{1}$, S. SONG ${ }^{1}$ \\ ${ }^{1}$ Department of Anesthesiology and Pain Medicine, School of Medicine, \\ Daegu Catholic University - Daegu (South Korea)
}

Background and Goal of Study: Left ventricular(LV) diastolic function does not recover from ischemia/reperfusion(IR) injury like acute myocardial infarction(MI) compared with systolic function and is important to surgical outcome under any type of anesthesia. Netrin-1 is known to improve IR injury at acute MI rat model. To evaluate the improvement of diastolic LV dysfunction as well as systolic function by preconditioning with netrin-1 after acute MI rat model, we examined the changes of echocardiographic parameters and compared with them before and after Ml using netin-1.
Materials and Methods:Male, 8-to 9-week-old, Spraque-Dawley rats with a mean body weight of $277.40 \pm 9.48 \mathrm{~g}$ were anesthesized with pentobarbital $(65 \mathrm{mg} / \mathrm{kg} \mathrm{IP})$ under intubation and positive pressure ventilation. The acute Ml model had the ligation of left coronary artery under heart exposure through 45 th intercostal space then closed. Another group of rat under went sham ligation without tightening the suture around the coronary artery. After 30 minutes of ischemia, netrin-1 $(5 \mathrm{mcg} / \mathrm{kg})$ was slowly injected into Ml group but vehicle(normal saline) into another MI group via tail vein. Using Vevo2100, Echocardiographic studies were performed before surgery and After 120 minutes of reperfusion. Echocardiographic parameters matched systolic function with fractional shortening(FS) and ejection fraction(EF), but diastolic function with $E^{\prime}$ and $E / E^{\prime}$ ratio.

\section{Control}
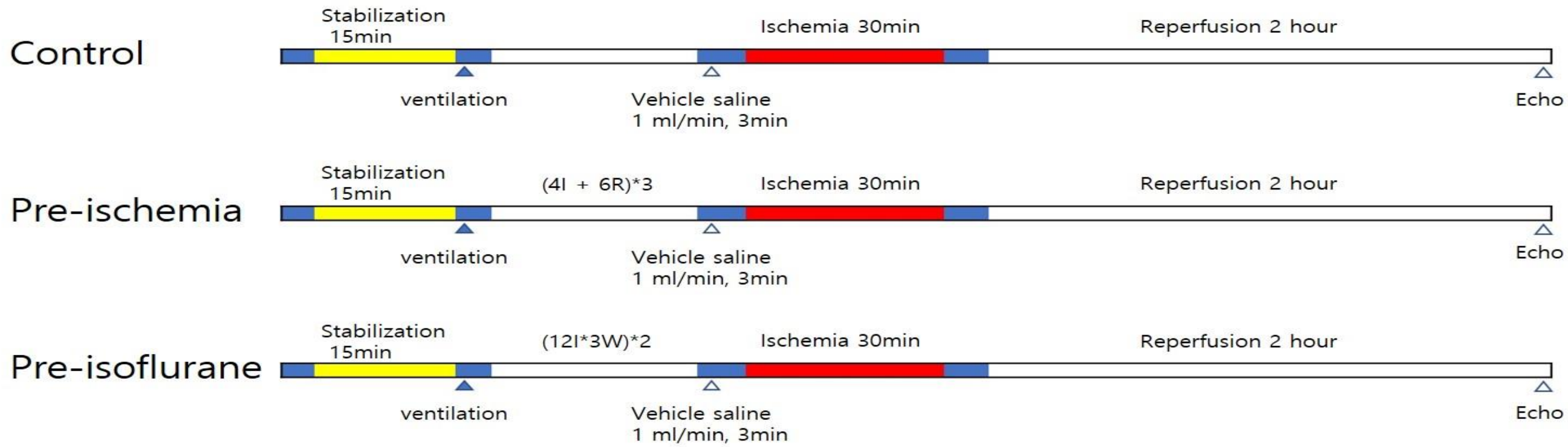

Netrin-1

\begin{tabular}{ccc}
$\begin{array}{c}\text { Stabilization } \\
15 \mathrm{~min}\end{array}$ & Ischemia 30min & \multicolumn{2}{c}{ Reperfusion 2 hour } \\
ventilation & $\begin{array}{c}\text { netrin-1 } \\
1 \mathrm{ml} / \mathrm{min}, 3 \mathrm{~min}\end{array}$ & $\Delta$ \\
Echo
\end{tabular}

Results and Discussion: MI-vehicle group had significantly attenuated FS, EF and E' compared with sham, while MI-netrin group had significant reduction in attenuation of them compared with MI-vehicle group. MI-vehicle group had significantly increased E/E' ratio compared with $\mathrm{MI}$-vehicle group, while $\mathrm{MI}$ netrin group had signifrcant reduction in increase of it.
Conclusion(s):Preconditioning with netrin-1 makes meaningful improvemet of systolic dysfunction with significant increase with FS and EF after acute MI. Also it helps E' recovery for LV diastolic function and E/E' ratio for left atrial pressure which means diastolic functional recovery.
$E^{\prime}$

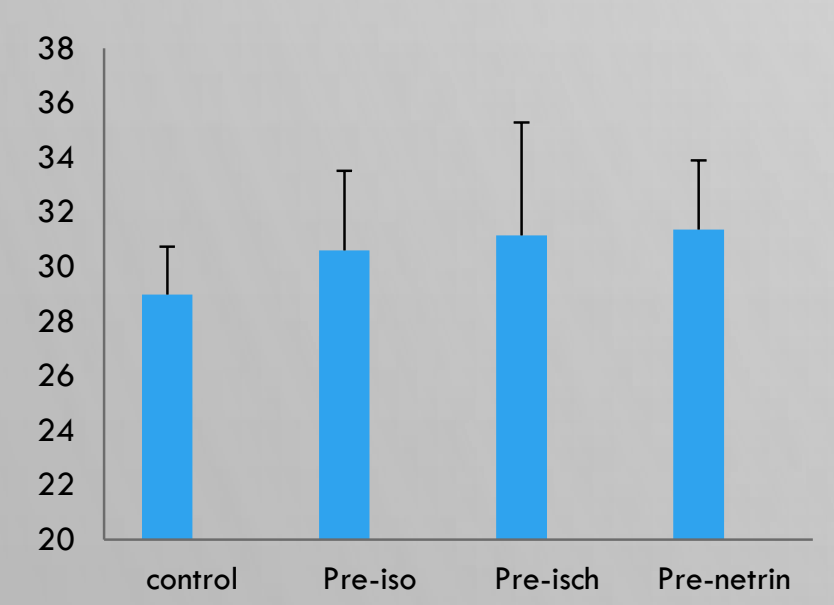

$E / E^{\prime}$

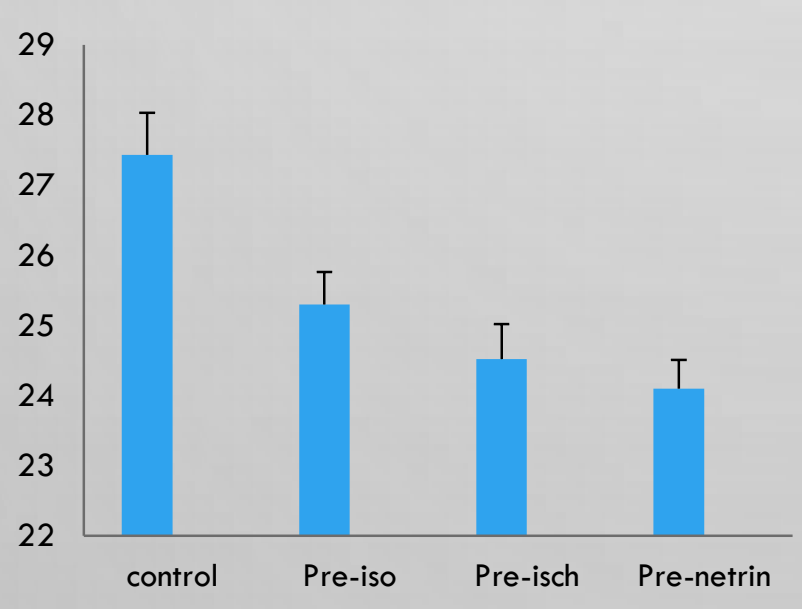

EF

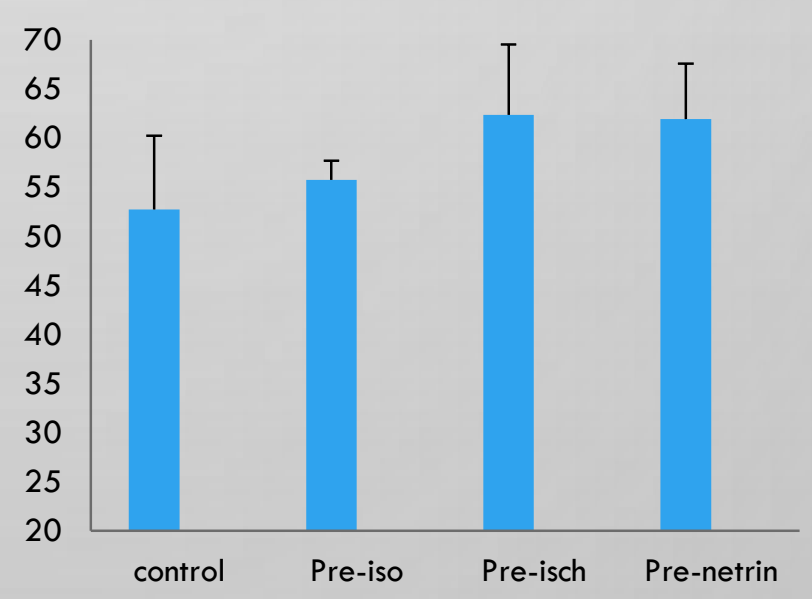

FS

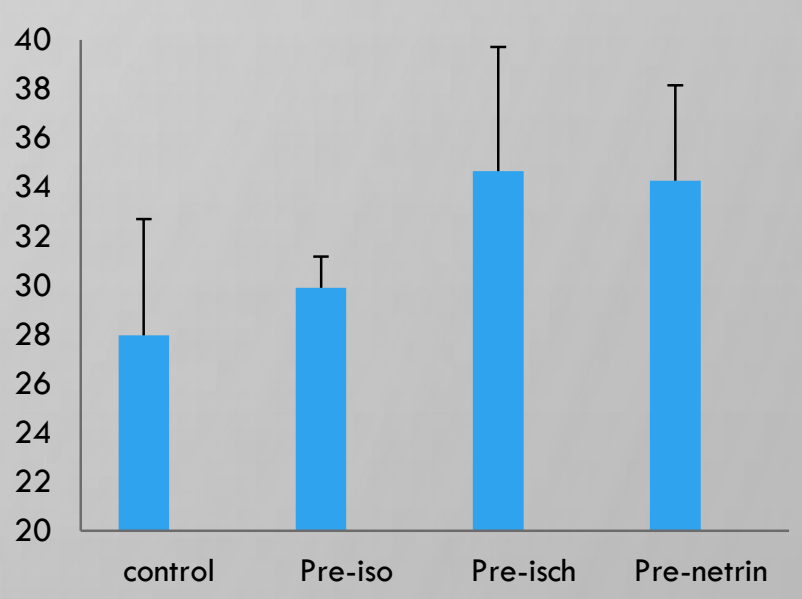

